OPEN ACCESS

Edited by:

Zheng Wang,

Shanghai Jiao Tong University, China

Reviewed by:

Zhichao Wang,

Shanghai Jiao Tong University, China

Wenjuan Wang,

Children's Hospital of Soochow

University, China

${ }^{*}$ Correspondence:

Zhian Li

sxeylza@163.com

Specialty section: This article was submitted to Women's Cancer,

a section of the journal

Frontiers in Oncology

Received: 15 November 2020 Accepted: 03 March 2021

Published: 18 March 2021

Citation:

Li Y and Li Z (2021) Potential Mechanism Underlying the

Role of Mitochondria in Breast Cancer Drug Resistance and Its

Related Treatment Prospects.

Front. Oncol. 11:629614. doi: 10.3389/fonc.2021.629614

\section{Potential Mechanism Underlying the Role of Mitochondria in Breast Cancer Drug Resistance and Its Related Treatment Prospects}

\author{
Yuefeng $L i$ and Zhian $L i^{*}$ \\ Department of Oncological Surgery, Shaoxing Second Hospital, Shaoxing, China
}

Breast cancer incidence and mortality rates have been consistently high among women. The use of diverse therapeutic strategies, including chemotherapy, endocrine therapy, targeted therapy, and immunotherapy, has improved breast cancer prognosis. However, drug resistance has become a tremendous obstacle in overcoming breast cancer recurrence and metastasis. It is known that mitochondria play an important role in carcinoma cell growth, invasion and apoptosis. Recent studies have explored the involvement of mitochondrial metabolism in breast cancer prognosis. Here, we will provide an overview of studies that investigated mitochondrial metabolism pathways in breast cancer treatment resistance, and discuss the application prospects of agents targeting mitochondrial pathways against drug-resistant breast cancer.

Keywords: breast cancer, mitochondrial, drug resistance, chemoresistance, tumor microenvironment

\section{INTRODUCTION}

Breast cancer is the second most common cancer in the world and ranks first in cancer incidence in women (1). Its diagnosis rate is increasing year by year, accompanied by a long-term high mortality rate (2). Early breast cancer is usually effectively treated using surgery alone or in combination with adjuvant radiotherapy. However, most patients with advanced breast cancer undergo mastectomy combined with radiotherapy and/or chemotherapy. Notably, the growing popularity of hormone therapy and targeted drug therapy in the treatment of breast cancer has greatly improved its fiveyear survival rate (3). Although breast cancer treatment methods have progressively diversified, the treatment of breast cancer, especially of triple-negative breast cancer(TNBC), a highly heterogeneous tumor, remains challenging (4), primarily due to chemotherapy resistance (5). Various recent studies have focused on discovering chemotherapy targets and the mechanisms underlying chemotherapy resistance to improve breast cancer prognosis. Furthermore, molecules, such as neuropilin-1 and follistatin-like 1 ,are considered to be involved in breast cancer resistance to doxorubicin. Nevertheless, the role of these new molecules in specific clinical applications requires further exploration. Therefore, there is an urgent need to explore more effective methods $(6,7)$.

Recently, the role of mitochondria in cancer has attracted increasing attention. It is well known that mitochondria play an important role in tumor cell occurrence, proliferation and apoptosis. Interestingly, recent studies have shown that tumor chemoresistance is closely related to mitochondria (8). Studies have demonstrated that mitochondrial fission is regulated by dynamin- 
related protein 1 (Drp-1) and mediated by high-mobility group box 1 protein (HMGB1), a chemotherapy-induced colon cancer product, promoting colorectal cancer tumor chemoresistance (9). Atovaquone, an antiparasitic drug, can effectively block mitochondrial respiration at clinically relevant concentrations, and further increase hepatocellular carcinoma chemosensitivity (10). These studies have laid the foundation for finding the treatments for chemoresistant cancers. In recent years, the role of mitochondria in breast cancer has received substantial attention $(11,12)$. However, the relationship between mitochondria and chemoresistance in breast cancer has not been systematically examined. This review focuses on studies that investigated the role of mitochondria in breast cancer chemoresistance and discusses the mechanisms and relevant treatment prospects.

\section{Mitochondrial in Tumor Metabolism and Breast Cancer Drug Resistance}

In the human body, tumor cells are found in a dynamic microenvironment, composed of complex stromal cells and extracellular matrix (13), in which mitochondria play an essential role. The original Warburg effect suggests that mitochondrial defects in tumor cells lead to impaired aerobic respiration, which renders tumor cells more prone to aerobic glycolytic metabolism $(14,15)$. It has been proposed that the changes in tumor cell metabolism are caused by the expression of oncogenes and hypoxia-related signal molecules that upregulate glycolytic enzymes. At the same time hypoxia inducible factor (HIF)-induced pyruvate dehydrogenase kinase (PDK) inhibits the PDH complex, and Akt, an oncogene, mediates the transcription of Glucose transporter type 1 (GLUT1) promoting the binding of hexokinase 2 to the voltage-dependent anion channels (VDAC) on the outer mitochondrial membrane to induce aerobic glycolysis. The synergistic effects of these pathways and the increase of mitochondrial autophagy lead to the glycolytic phenotype of tumor cells $(16,17)$. On the other hand, a series of studies on the "reverse" Warburg effect revealed that cancerassociated fibroblasts in the tumor microenvironment can change their phenotype through mitochondrial dysfunction and aerobic glycolysis, thereby providing high-energy nutrients to tumor epithelial cells promoting tumor growth, metastasis and chemoresistance $(15,18,19)$. Furthermore, Sotgia, F et al. found that 15 molecular markers related to mitochondrial germination and translation were differentially expressed in the tumor microenvironment using an analysis of genome-wide transcription profile data of human breast cancer cells and immunohistochemical verification. In addition, the important role of mitochondria in the metabolic symbiosis between tumor epithelial cancer cells and their surrounding stroma, suggests that the targeting mitochondrial gene expression and translation may be a new treatment approach for breast cancer (20).

The complex metabolic network of the tumor microenvironment is regulated by a variety of molecules, many of which have been found to participate in mitochondria-related metabolic pathways $(21,22)$. Caveolin-1(CAV-1) is a major structural protein in small plasma membrane invaginations that maintains membrane stability and signal transduction (23). Clinical studies have shown that CAV-1 is an important predictor of breast cancer prognosis $(24,25)$. Furthermore, it has been found that a decrease in CAV-1 levels in stromal cells in the tumor microenvironment enhances breast cancer resistance to tamoxifen (24). Moreover, in a study using mouse xenograft models, it was found that CAV-1 expression was positively correlated with the tumor sensitivity to nab-paclitaxel (26). CAV-1 downregulation in tumor-associated stromal fibroblasts could increase reactive oxygen species (ROS) production, thus inducing oxidative stress followed by autophagy and mitochondrial dysfunction. The outcome of this metabolic change will promote mitochondrial metabolism using high-energy substances, such as L-lactic acid and ketone bodies to provide nourishment to epithelial cancer cells. The mitochondrialtargeted superoxide dismutase 2(SOD2) is a potential inhibitor that can resist to oxidative stress and block the production of molecules that can nourish tumors, thereby effectively reversing the tumorpromoting phenotype of CAV-1 in breast cancer cells (27). Monocarboxylate transporter 4 (MCT4), an independent prognostic factor for breast cancer survival, was found to be negatively correlated with the expression of CAV-1 $(28,29)$. Thus, the combined analysis of MCT4 and CAV-1 expression levels in the matrix can improve the accuracy of breast cancer prognosis. The antioxidant $\mathrm{N}$-acetyl-cysteine has been shown to inhibit the oxidative stress-induced formation of MCT4. Furthermore, MCT4 inhibitors can effectively inhibit the influx of L-lactic acid and ketone bodies into the tumor microenvironment, constituting a novel strategy for tumor treatment. However, MCT1 inhibitors are currently considered to have similar efficacy to that of MCT4 and are employed in the clinical studies $(29,30)$. Corresponding to these findings, CAV-1 was found to be overexpressed in drug-resistant breast cancer cells (31). Astragaloside IV (AS-IV), a biologically active substance purified from Astragalus, can work synergistically with paclitaxel to trigger the mitochondrial apoptosis pathway and effectively induce drugresistant breast cancer cell death. This process involves AS-IV activation of eNOS/NO/ONOO ${ }^{-}$signaling by $\mathrm{CAV}-1$ inhibition that enhances the chemosensitivity of breast cancer cells to paclitaxel (32) (Figure 1). Intriguingly, the expression level of CAV-1 in TNBC has also been found to be negatively correlated with cancer cell radiation sensitivity (33).

\section{Mitochondria in Tumor Apoptosis and Breast Cancer Drug Resistance}

B-cell lymphoma 2 (BCL-2) family proteins play a crucial role in the process of regulating cell apoptosis and have both proapoptotic and anti-apoptotic activities (34). Typical pro- and anti-apoptotic members are BAX, BCL2, and BCL-XL, respectively $(35,36)$. Pro-apoptotic proteins such as BAX can promote the release of cytochrome $\mathrm{C}$ and second mitochondriaderived activator of caspases (Smac) from mitochondria, leading to cysteinyl aspartate specific proteinase (caspase)-induced cell apoptosis. BCL-2 and BCL-XL inhibit the pro-apoptotic effects of BAX and other molecules (37). In this way, the anti-apoptotic BCL-2 family proteins help breast tumor cells escape apoptosis and acquire drug resistance (38). Thus, inhibiting anti-apoptotic BCL-2 family proteins is a potentially valuable therapeutic strategy against breast cancer drug resistance. Studies have 


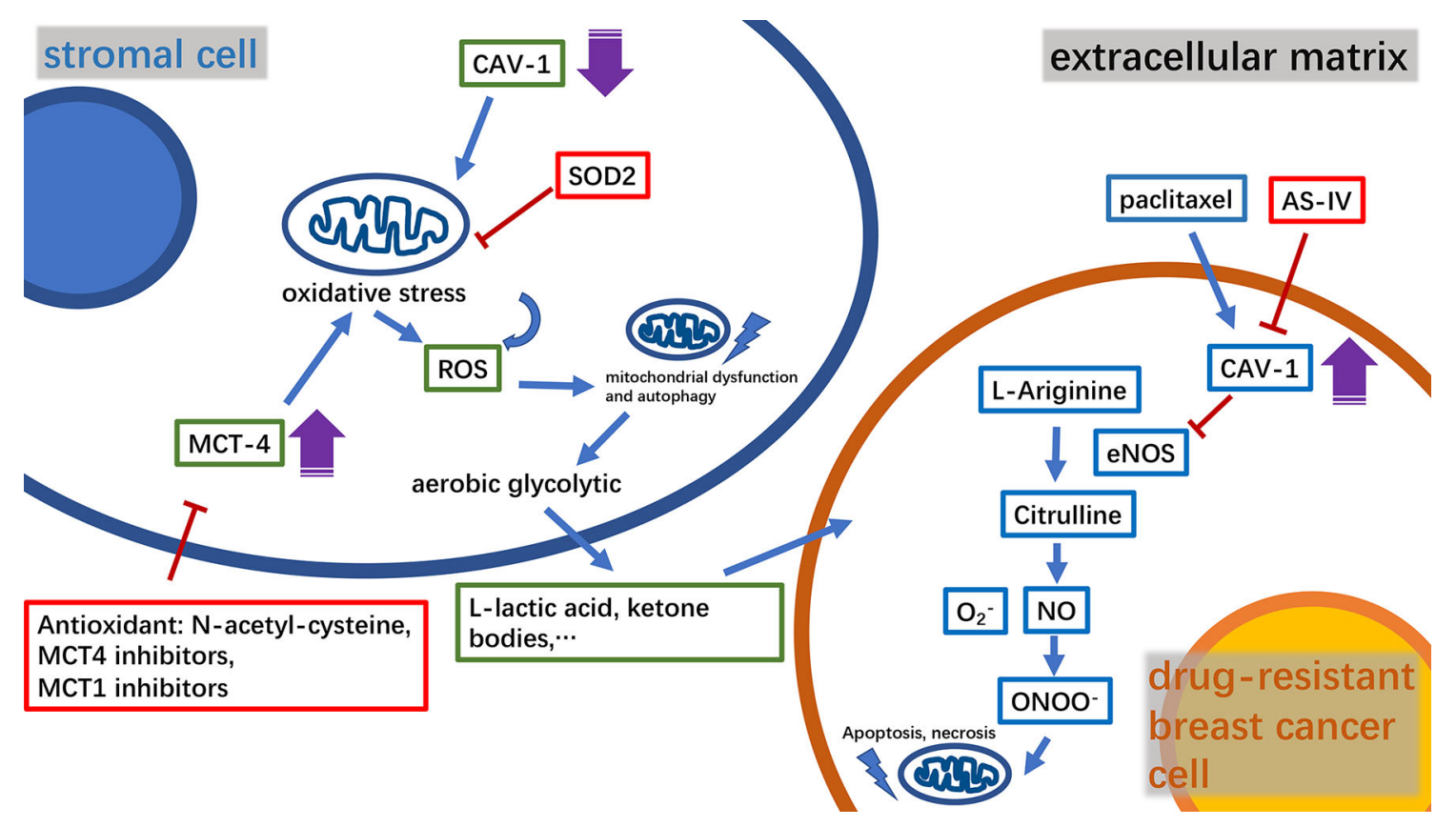

FIGURE 1 | A diagram of the relationship between key molecules in the microenvironment of drug-resistant breast cancer tumors and mitochondria, and the mechanism of the relevant drugs. CAV-1, caveolin-1; MCT4, monocarboxylate transporter 4; ROS, reactive oxygen species; SOD2, superoxide dismutase 2; AS-IV, astragaloside IV; NO, nitric oxide.

found that microRNA-195 can target BCL-2 to trigger mitochondrial dysfunction and then cause apoptosis, thereby enhancing the therapeutic efficacy of the chemotherapy drug etoposide in breast cancer (39). Via another mechanism, Sabutoclax (BI-97C1), a BCL-2 homology domain 3 (BH3) mimetic, acts as a pan-BCL-2 inhibitor (40). Sabutoclax has shown potent cytotoxicity against drug-resistant breast cancer in vivo and in vitro. Furthermore, Sabutoclax not only causes the release of caspase from mitochondria to cause cancer cell apoptosis, but also blocks the interleukin 6/signal transducers and activators of transcription (IL-6/STAT) pathway to eliminate the breast cancer stem cells. Interestingly, it has also been successfully used in combination with standard chemotherapy to treat chemoresistant breast cancer (41). Consistent with their principle of action, drugs, such as ABT737, ABT-263 (Navitoclax) and $\alpha$-tocopheryl succinate ( $\alpha$-TOS), target BCL-2 in the mitochondria (Figure 2). Among them, ABT-737 has been confirmed to improve the docetaxel resistance in TNBC cell lines overexpressing BCL-2 using cytology experiments. Furthermore, using a new technology the drug is encapsulated in poly lactic-co-glycolic acid nanoparticles (NPs) to accumulate drugs in xenograft TNBC tumors and exert effective anti-tumor effects, thus providing a good foundation for successful drug targeting to human breast tumors in the future to avoid systemic side effects $(42,43)$. It is worth mentioning that Navitoclax has passed phase I of a clinical trial in refractory chronic lymphocytic leukemia, and the optimal drug concentration has also been further explored in a phase II clinical trial. In breast cancer treatment, Navitoclax has been proven to enhance the effectiveness of epidermal growth factor receptor (EGFR)-targeted antibody-drug conjugates for TNBC treatment in animal experiments. In addition, recent studies have also revealed the inherent drug resistance of TNBC to Navitoclax. Therefore, it is necessary to evaluate the specific value of the drug in conjunction with TNBC genomic research (44-46). The combination of $\alpha$-TOS and high-dose tamoxifen can effectively inhibit the proliferation activity of TNBC, and improve the anti-cancer effect of pterostilbene in breast cancer xenograft mice. In a recent study, pluronic polymer (P123) was modified into ortho ester End-capping (P123-OE) and bridged with a-TOS to form a copolymer (POT), and then doxorubicin-loaded POT micelles (POT-DOX) were used in breast cancer animal models, which effectively increased the accumulation of drugs in multi-drug-resistant breast cancer cells and enhanced the drug's anti-cancer effects, potentially providing alternative clinical treatment options (47-49). Myeloid cell leukemia-1 (MCL1) is another typical antiapoptotic protein belonging to the BCL-2 family (50). The myelocytomatosis oncogene (MYC), a proto-oncogene, encodes a transcription factor involved in cancer cell proliferation and apoptosis $(51,52)$. The mRNA and protein levels of these two molecules were co-amplified in paclitaxel-resistant breast cancer cell lines. MYC and MCL-1 mediate the enrichment of breast cancer stem cells(CSCs) $(53,54)$. Among them, MCL2 has also been confirmed to be located in the mitochondrial matrix to enhance mitochondrial oxidative phosphorylation (mtOXPHOS), which in turn increases the ROS generation, activates hypoxia stress, and causes drug resistance-mediated 


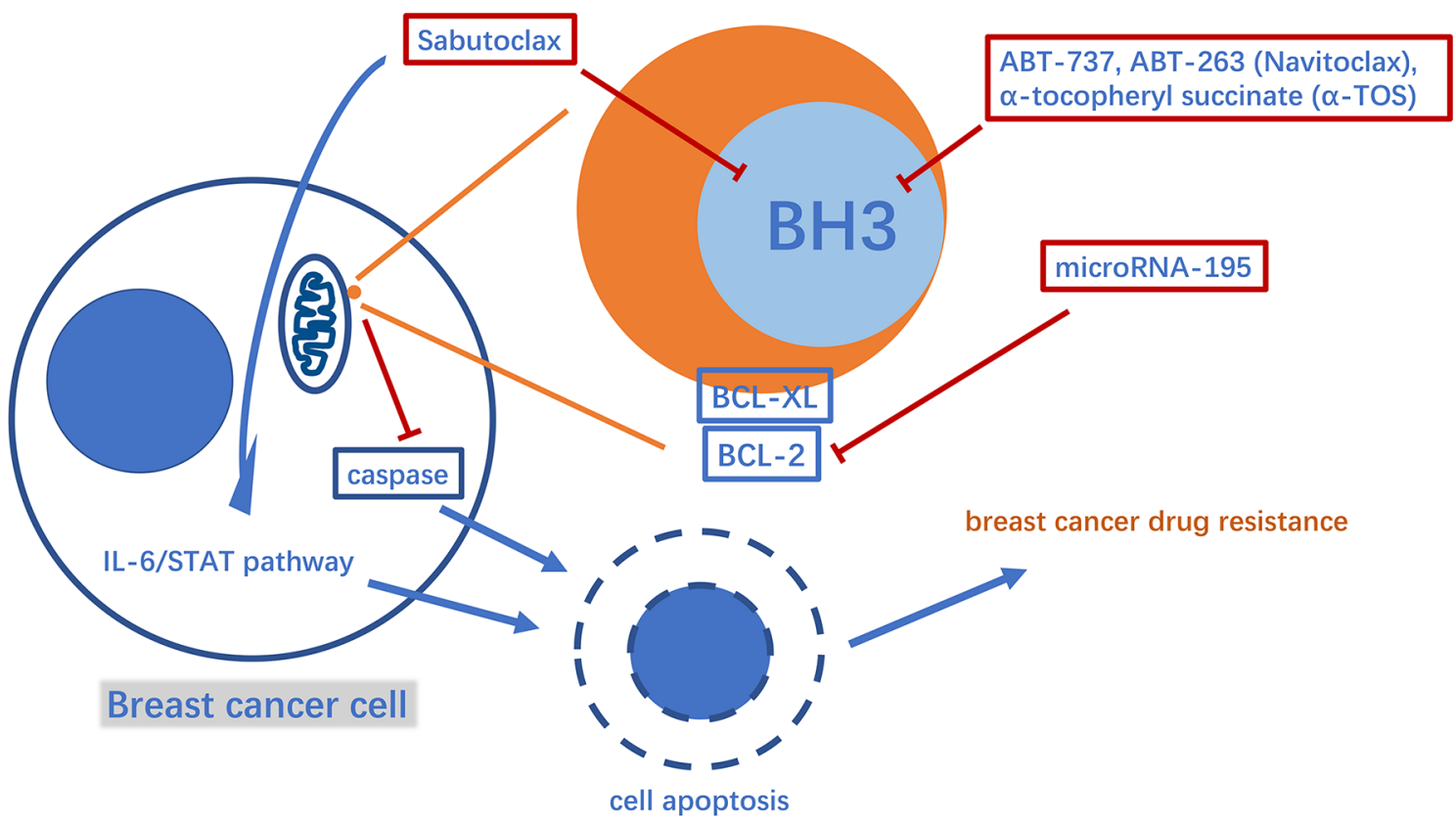

FIGURE 2 | A diagram of some mechanism of mitochondria involved in apoptosis-mediated breast cancer drug resistance and related drugs. BH3, BCL-2 homology domain 3.

CSC enrichment $(53,55)$. Therefore, drugs that inhibit HIF-1a, such as $\mathrm{N}$-acetylcysteine, oligomycin and digoxin, may provide direction in the treatment of drug-resistant breast cancer (56-58).

\section{Mitochondrial Dynamics and Breast Cancer Drug Resistance}

Mitochondria are highly dynamic organelles that change their shape, size, and distribution to adapt to changes in different cell states via the coordinate action of fission and fusion (59). Dynaminrelated protein 1 (Drp1), a classical mitochondrial fission protein, can cause mitochondria to divide into two to form a small circular mitochondrial fragment network. Mitochondrial fusion protein (MFN) and optic atrophy 1(OPA1), can elongate or cluster together through fusion (59-61). A study of tamoxifen-resistant breast cell lines found that the drug-resistant cell lines have a more fragmented mitochondrial network. In these cell lines, serine 637, an essential phosphorylation site of DRP1, was activated, while serine 616 , another vital phosphorylation site, was not, which increased the mitochondrial fission activity of DRP1, causing mitochondrial fragmentation. Based on this, changes in mitochondrial dynamics that are closely related to breast cancer drug-resistance can be improved (62). Current research on drugs targeting mitochondrial dynamics is mostly focused on breast cancer growth and proliferation rather than breast cancer drug resistance (63-65). Mitochondrial transplantation is a new type of biological technology that gradually extends from animal models to human clinical applications (66). Using this technology, exogenous healthy mitochondria are transplanted into cells with damaged mitochondria to achieve the treatment purpose (67). A study on mitochondrial transplantation in breast cancer cell lines found that the protein levels of MFN2 and OPA1 in the cells significantly increased after the transplantation of exogenous healthy mitochondria into breast cancer cells, while the protein level of drp1 dramatically decreased. Interestingly, the morphology of the mitochondria in the cell was mostly elongated to tubular, while the fragmented mitochondria were obviously inhibited. In addition, the resistance of breast cancer cells to the anticancer drugs doxorubicin and paclitaxel was also significantly reduced (68). Recent studies have also suggested that mitochondrial transplantation can change the tumor microenvironment to combat breast cancer, and demonstrated the therapeutic effect of mitochondrial transplantation on breast cancer in animal models (69). In these studies, we observed that while mitochondrial transplantation brings about changes in mitochondrial morphology, it also regulates functions, such as oxidative respiration. The morphology and function of mitochondria are inextricably linked $(68,69)$. Mitochondrial transplantation is a new approach for the treatment of drug-resistant breast cancer.

\section{Mitochondrial DNA and Breast Cancer Drug-Resistance}

Mitochondrial DNA(mtDNA), a double-stranded circular DNA, contains 37 genes encoding rRNA, tRNA, and oxidative phosphorylation complex-related proteins (70). A variety of treatments that target mitochondrial gene expression have been explored in a variety of diseases, including breast cancer, with proven therapeutic benefits (71). The link between mitochondrial gene copy number and breast cancer treatment resistance has attracted increasing attention $(72,73)$. Metformin 
can stunt breast cancer progress by inhibiting complex I encoded by the electron transport chain gene in mtDNA $(74,75)$. The decrease in $\mathrm{BTB}$ and $\mathrm{CNC}$ homology 1(BACH1), a heminbinding transcription factor, can promote the expression of the electron transport chain(ETC) genes to increase the sensitivity of breast cancer to metformin. The specific degradation of BACH1 by panhematin (an FDA-approved drug) can effectively enhance the sensitivity of breast cancer cells to metformin treatment in vitro and in vivo (76). Not long ago, another extraordinary study found that cancer-related fibroblast-derived exosomes with complete genomic mitochondrial DNA can be obtained by breast stem cell-like cancer cells and display mtOXPHOSdependent breast cancer endocrine-resistant therapy (77). Hitherto, drugs that target mtDNA, such as vitamin K3 (menadione), are often used in combination with other drugs in the treatment of breast cancer $(78,79)$. However, whether they improve resistance to breast cancer treatment remains unclear. This is worth exploring in future studies.

\section{CONCLUSIONS}

The problem of drug resistance in the comprehensive treatment model of breast cancer has been extensively investigated, and mitochondria have been found to play a subtle role in the process of breast cancer drug resistance. Exploration of the role of mitochondria in breast cancer drug resistance in the tumor microenvironment and cancer cell interior indicates that: 1) numerous molecules in the tumor microenvironment can mediate the production of a variety of metabolites to induce drug resistance through the action of mitochondria, 2) mitochondria can regulate cell apoptosis and affect breast cancer resistance, 3) morphological and functional changes in the mitochondria can promote breast cancer resistance, and 4) the expression level of mtDNA can mediate breast cancer resistance. These studies have proposed the use of effective molecular targeted drugs or new treatments to sensitize breast cancer cells to drugs, and some drugs are already used in clinical research. Exploring the role of mitochondria in breast cancer chemoresistance is expected to open up novel ways for breast cancer treatment.

\section{AUTHOR CONTRIBUTIONS}

YL: literature search, writing, and editing of the manuscript. ZL: Analysis, design, writing-review and edit, and supervision. All authors contributed to the article and approved the submitted version.

\section{REFERENCES}

1. Bray F, Ferlay J, Soerjomataram I, Siegel RL, Torre LA, Jemal A. Global cancer statistics 2018: GLOBOCAN estimates of incidence and mortality worldwide for 36 cancers in 185 countries. CA: Cancer J Clin (2018) 68:394-424. doi: $10.3322 /$ caac. 21492

2. Siegel RL, Miller KD, Jemal A. Cancer statistics, 2019. CA: Cancer J Clin (2019) 69:7-34. doi: 10.3322/caac.21551

3. Miller KD, Nogueira L, Mariotto AB, Rowland JH, Yabroff KR, Alfano CM, et al. Cancer treatment and survivorship statistics, 2019. CA: Cancer J Clin (2019) 69:363-85. doi: 10.3322/caac.21565

4. Chang-Qing Y, Jie L, Shi-Qi Z, Kun Z, Zi-Qian G, Ran X, et al. Recent treatment progress of triple negative breast cancer. Prog Biophys Mol Biol (2020) 151:40-53. doi: 10.1016/j.pbiomolbio.2019.11.007

5. Kim C, Gao R, Sei E, Brandt R, Hartman J, Hatschek T, et al. Chemoresistance Evolution in Triple-Negative Breast Cancer Delineated by Single-Cell Sequencing. Cell (2018) 173:879-93.e13. doi: 10.1016/j.cell.2018.03.041

6. Naik A, Al-Yahyaee A, Abdullah N, Sam J-E, Al-Zeheimi N, Yaish MW, et al. Neuropilin-1 promotes the oncogenic Tenascin-C/integrin $\beta 3$ pathway and modulates chemoresistance in breast cancer cells. BMC Cancer (2018) 18:533. doi: 10.1186/s12885-018-4446-y

7. Cheng S, Huang Y, Lou C, He Y, Zhang Y, Zhang Q. FSTL1 enhances chemoresistance and maintains stemness in breast cancer cells via integrin $\beta 3 /$ Wnt signaling under miR-137 regulation. Cancer Biol Ther (2019) 20:32837. doi: 10.1080/15384047.2018.1529101

8. Guerra F, Arbini AA, Moro L. Mitochondria and cancer chemoresistance. Biochim Biophys Acta Bioenerg (2017) 1858:686-99. doi: 10.1016/j.bbabio.2017.01.012

9. Huang C-Y, Chiang S-F, Chen WT-L, Ke T-W, Chen T-W, You Y-S, et al. HMGB1 promotes ERK-mediated mitochondrial Drp1 phosphorylation for chemoresistance through RAGE in colorectal cancer. Cell Death Dis (2018) 9:1004. doi: 10.1038/s41419-018-1019-6

10. Sun $\mathrm{Y}, \mathrm{Xu} \mathrm{H}$, Chen X, Li X, Luo B. Inhibition of mitochondrial respiration overcomes hepatocellular carcinoma chemoresistance. Biochem Biophys Res Commun (2019) 508:626-32. doi: 10.1016/j.bbrc.2018.11.182

11. Porporato PE, Filigheddu N, Pedro JMB, Kroemer G, Galluzzi L. Mitochondrial metabolism and cancer. Cell Res (2018) 28:265-80. doi: $10.1038 /$ cr.2017.155

12. Gururaja Rao S. Mitochondrial Changes in Cancer. Handb Exp Pharmacol (2017) 240:211-27. doi: 10.1007/164_2016_40

13. Sotgia F, Martinez-Outschoorn UE, Howell A, Pestell RG, Pavlides S, Lisanti MP. Caveolin-1 and cancer metabolism in the tumor microenvironment: markers, models, and mechanisms. Annu Rev Pathol (2012) 7:423-67. doi: 10.1146/annurev-pathol-011811-120856

14. Vander Heiden MG, Cantley LC, Thompson CB. Understanding the Warburg effect: the metabolic requirements of cell proliferation. Science (2009) 324:1029-33. doi: 10.1126/science.1160809

15. Sotgia F, Martinez-Outschoorn UE, Pavlides S, Howell A, Pestell RG, Lisanti MP. Understanding the Warburg effect and the prognostic value of stromal caveolin-1 as a marker of a lethal tumor microenvironment. Breast Cancer Res (2011) 13:213. doi: 10.1186/bcr2892

16. Smolkova K, Plecita-Hlavata L, Bellance N, Benard G, Rossignol R, Jezek P. Waves of gene regulation suppress and then restore oxidative phosphorylation in cancer cells. Int J Biochem Cell Biol (2011) 43:950-68. doi: 10.1016/ j.biocel.2010.05.003

17. Pedersen PL. Warburg, me and Hexokinase 2: Multiple discoveries of key molecular events underlying one of cancers' most common phenotypes, the "Warburg Effect", i.e., elevated glycolysis in the presence of oxygen. J Bioenerg Biomembr (2007) 39:211-22. doi: 10.1007/s10863-007-9094-x

18. Balliet RM, Capparelli C, Guido C, Pestell TG, Martinez-Outschoorn UE, Lin $\mathrm{Z}$, et al. Mitochondrial oxidative stress in cancer-associated fibroblasts drives lactate production, promoting breast cancer tumor growth. Cell Cycle (2014) 10:4065-73. doi: 10.4161/cc.10.23.18254

19. Martinez-Outschoorn UE, Sotgia F, Lisanti MP. Power surge: supporting cells "fuel" cancer cell mitochondria. Cell Metab (2012) 15:4-5. doi: 10.1016/ j.cmet.2011.12.011

20. Sotgia F, Whitaker-Menezes D, Martinez-Outschoorn UE, Salem AF, Tsirigos A, Lamb R, et al. Mitochondria "fuel" breast cancer metabolism: fifteen markers of mitochondrial biogenesis label epithelial cancer cells, but are 
excluded from adjacent stromal cells. Cell Cycle (2012) 11:4390-401. doi: $10.4161 / \mathrm{cc} .22777$

21. Hu M, Yao J, Cai L, Bachman KE, F. van den Brule V, Velculescu V, et al. Distinct epigenetic changes in the stromal cells of breast cancers. Nat Genet (2005) 37:899-905. doi: 10.1038/ng1596

22. Martinez-Outschoorn U, Sotgia F, Lisanti MP. Tumor microenvironment and metabolic synergy in breast cancers: critical importance of mitochondrial fuels and function. Semin Oncol (2014) 41:195-216. doi: 10.1053/j.seminoncol.2014.03.002

23. Cheng JPX, Nichols BJ. Caveolae: One Function or Many? Trends Cell Biol (2016) 26:177-89. doi: 10.1016/j.tcb.2015.10.010

24. El-Gendi SM, Mostafa MF, El-Gendi AM. Stromal caveolin-1 expression in breast carcinoma. Correlation with early tumor recurrence and clinical outcome. Pathol Oncol Res (2012) 18:459-69. doi: 10.1007/s12253-0119469-5

25. Witkiewicz AK, Dasgupta A, Sammons S, Er O, Potoczek MB, Guiles F, et al. Loss of stromal caveolin-1 expression predicts poor clinical outcome in triple negative and basal-like breast cancers. Cancer Biol Ther (2010) 10:135-43. doi: $10.4161 /$ cbt.10.2.11983

26. Chatterjee M, Ben-Josef E, Robb R, Vedaie M, Seum S, Thirumoorthy K, et al. Caveolae-Mediated Endocytosis Is Critical for Albumin Cellular Uptake and Response to Albumin-Bound Chemotherapy. Cancer Res (2017) 77:5925-37. doi: 10.1158/0008-5472.CAN-17-0604

27. Trimmer C, Sotgia F, Whitaker-Menezes D, Balliet RM, Eaton G, MartinezOutschoorn UE, et al. Caveolin-1 and mitochondrial SOD2 (MnSOD) function as tumor suppressors in the stromal microenvironment: a new genetically tractable model for human cancer associated fibroblasts. Cancer Biol Ther (2011) 11:383-94. doi: 10.4161/cbt.11.4.14101

28. Doyen J, Trastour C, Ettore F, Peyrottes I, Toussant N, Gal J, et al. Expression of the hypoxia-inducible monocarboxylate transporter MCT4 is increased in triple negative breast cancer and correlates independently with clinical outcome. Biochem Biophys Res Commun (2014) 451:54-61. doi: 10.1016/ j.bbrc.2014.07.050

29. Witkiewicz AK, Whitaker-Menezes D, Dasgupta A, Philp NJ, Lin Z, Gandara R, et al. Using the "reverse Warburg effect" to identify high-risk breast cancer patients: stromal MCT4 predicts poor clinical outcome in triple-negative breast cancers. Cell Cycle (2012) 11:1108-17. doi: 10.4161/cc.11.6.19530

30. Whitaker-Menezes D, Martinez-Outschoorn UE, Lin Z, Ertel A, Flomenberg N, Witkiewicz AK, et al. Evidence for a stromal-epithelial "lactate shuttle" in human tumors: MCT4 is a marker of oxidative stress in cancer-associated fibroblasts. Cell Cycle (2011) 10:1772-83. doi: 10.4161/cc.10.11.15659

31. Wang Z, Wang N, Li W, Liu P, Chen Q, Situ H, et al. Caveolin-1 mediates chemoresistance in breast cancer stem cells via $\beta$-catenin/ABCG2 signaling pathway. Carcinogenesis (2014) 35:2346-56. doi: 10.1093/carcin/bgu155

32. Zheng Y, Dai Y, Liu W, Wang N, Cai Y, Wang S, et al. Astragaloside IV enhances taxol chemosensitivity of breast cancer via caveolin-1-targeting oxidant damage. J Cell Physiol (2019) 234:4277-90. doi: 10.1002/jcp.27196

33. Zou M, Li Y, Xia S, Chu Q, Xiao X, Qiu H, et al. Knockdown of CAVEOLIN-1 Sensitizes Human Basal-Like Triple-Negative Breast Cancer Cells to Radiation. Cell Physiol Biochem (2017) 44:778-91. doi: 10.1159/000485291

34. Wang NS, Unkila MT, Reineks EZ, Distelhorst CW. Transient expression of wild-type or mitochondrially targeted $\mathrm{Bcl}-2$ induces apoptosis, whereas transient expression of endoplasmic reticulum-targeted $\mathrm{Bcl}-2$ is protective against Bax-induced cell death. J Biol Chem (2001) 276:44117-28. doi: $10.1074 /$ jbc.M101958200

35. Oltvai ZN, Milliman CL, Korsmeyer SJ. Bcl-2 heterodimerizes in vivo with a conserved homolog, Bax, that accelerates programmed cell death. Cell (1993) 74:609-19. doi: 10.1016/0092-8674(93)90509-O

36. Boise LH, González-García M, Postema CE, Ding L, Lindsten T, Turka LA, et al. bcl-x, a bcl-2-related gene that functions as a dominant regulator of apoptotic cell death. Cell (1993) 74:597-608. doi: 10.1016/0092-8674(93)90508-N

37. Bai L, Wang S. Targeting apoptosis pathways for new cancer therapeutics. Annu Rev Med (2014) 65:139-55. doi: 10.1146/annurev-med-010713-141310

38. Emi M, Kim R, Tanabe K, Uchida Y, Toge T. Targeted therapy against Bcl-2related proteins in breast cancer cells. Breast Cancer Res (2005) 7:R940-52. doi: $10.1186 / \mathrm{bcr} 1323$

39. Purohit PK, Edwards R, Tokatlidis K, Saini N. MiR-195 regulates mitochondrial function by targeting mitofusin-2 in breast cancer cells. RNA Biol (2019) 16:918-29. doi: 10.1080/15476286.2019.1600999
40. Goff DJ, Court Recart A, Sadarangani A, Chun HJ, Barrett CL, Krajewska M, et al. A Pan-BCL2 inhibitor renders bone-marrow-resident human leukemia stem cells sensitive to tyrosine kinase inhibition. Cell Stem Cell (2013) 12:31628. doi: 10.1016/j.stem.2012.12.011

41. Hu Y, Yagüe E, Zhao J, Wang L, Bai J, Yang Q, et al. Sabutoclax, pan-active BCL-2 protein family antagonist, overcomes drug resistance and eliminates cancer stem cells in breast cancer. Cancer Lett (2018) 423:47-59. doi: 10.1016/ j.canlet.2018.02.036

42. Hwang E, Hwang SH, Kim J, Park JH, Oh S, Kim YA, et al. ABT-737 ameliorates docetaxel resistance in triple negative breast cancer cell line. Ann Surg Treat Res (2018) 95:240-8. doi: 10.4174/astr.2018.95.5.240

43. Valcourt DM, Dang MN, Scully MA, Day ES. Nanoparticle-Mediated CoDelivery of Notch-1 Antibodies and ABT-737 as a Potent Treatment Strategy for Triple-Negative Breast Cancer. ACS Nano (2020) 14:3378-88. doi: 10.1021/acsnano.9b09263

44. Roberts AW, Seymour JF, Brown JR, Wierda WG, Kipps TJ, Khaw SL, et al. Substantial susceptibility of chronic lymphocytic leukemia to BCL2 inhibition: results of a phase I study of navitoclax in patients with relapsed or refractory disease. J Clin Oncol (2012) 30:488-96. doi: 10.1200/JCO.2011.34.7898

45. Zoeller JJ, Vagodny A, Daniels VW, Taneja K, Tan BY, DeRose YS, et al. Navitoclax enhances the effectiveness of EGFR-targeted antibody-drug conjugates in PDX models of EGFR-expressing triple-negative breast cancer. Breast Cancer Res (2020) 22:132. doi: 10.1186/s13058-020-01374-8

46. Marczyk M, Patwardhan GA, Zhao J, Qu R, Li X, Wali VB, et al. Multi-Omics Investigation of Innate Navitoclax Resistance in Triple-Negative Breast Cancer Cells. Cancers (Basel) (2020) 12:2551. doi: 10.3390/cancers12092551

47. Wei CW, Yu YL, Chen YH, Hung YT, Yiang GT. Anticancer effects of methotrexate in combination with alphatocopherol and alphatocopherol succinate on triplenegative breast cancer. Oncol Rep (2019) 41:2060-6. doi: 10.3892/or.2019.6958

48. Tam KW, Ho CT, Tu SH, Lee WJ, Huang CS, Chen CS, et al. $\alpha$-Tocopherol succinate enhances pterostilbene anti-tumor activity in human breast cancer cells in vivo and in vitro. Oncotarget (2018) 9:4593-606. doi: 10.18632/ oncotarget. 23390

49. Cheng X, Zeng X, Zheng Y, Fang Q, Wang X, Wang J, et al. pH-sensitive pluronic micelles combined with oxidative stress amplification for enhancing multidrug resistance breast cancer therapy. J Colloid Interface Sci (2020) 565:254-69. doi: 10.1016/j.jcis.2020.01.029

50. Fletcher S. MCL-1 inhibitors - where are we now (2019)? Expert Opin Ther Pat (2019) 29:909-19. doi: 10.1080/13543776.2019.1672661

51. Gnanaprakasam JN, Wang R. MYC in Regulating Immunity: Metabolism and Beyond. Genes (Basel) (2017) 8:88. doi: 10.3390/genes8030088

52. Dang CV. MYC on the path to cancer. Cell (2012) 149:22-35. doi: 10.1016/ j.cell.2012.03.003

53. Lee KM, Giltnane JM, Balko JM, Schwarz LJ, Guerrero-Zotano AL, Hutchinson KE, et al. MYC and MCL1 Cooperatively Promote Chemotherapy-Resistant Breast Cancer Stem Cells via Regulation of Mitochondrial Oxidative Phosphorylation. Cell Metab (2017) 26:633-47.e7. doi: 10.1158/1538-7445.AM2016-3328

54. Beck B, Blanpain C. Unravelling cancer stem cell potential. Nat Rev Cancer (2013) 13:727-38. doi: 10.1038/nrc3597

55. Perciavalle RM, Stewart DP, Koss B, Lynch J, Milasta S, Bathina M, et al. Antiapoptotic MCL-1 localizes to the mitochondrial matrix and couples mitochondrial fusion to respiration. Nat Cell Biol (2012) 14:575-83. doi: $10.1038 / \mathrm{ncb} 2488$

56. Zhang H, Qian DZ, Tan YS, Lee K, Gao P, Ren YR, et al. Digoxin and other cardiac glycosides inhibit HIF-1alpha synthesis and block tumor growth. Proc Natl Acad Sci U S A (2008) 105:19579-86. doi: 10.1073/pnas.0809763105

57. Samanta D, Gilkes DM, Chaturvedi P, Xiang L, Semenza GL. Hypoxiainducible factors are required for chemotherapy resistance of breast cancer stem cells. Proc Natl Acad Sci U S A (2014) 111:E5429-38. doi: 10.1073/ pnas. 1421438111

58. Gong Y, Agani FH. Oligomycin inhibits HIF-1alpha expression in hypoxic tumor cells. Am J Physiol Cell Physiol (2005) 288:C1023-9. doi: 10.1152/ ajpcell.00443.2004

59. Tilokani L, Nagashima S, Paupe V, Prudent J. Mitochondrial dynamics: overview of molecular mechanisms. Essays Biochem (2018) 62:341-60. doi: 10.1042/EBC20170104 
60. Zhao J, Zhang J, Yu M, Xie Y, Huang Y, Wolff DW, et al. Mitochondrial dynamics regulates migration and invasion of breast cancer cells. Oncogene (2013) 32:4814-24. doi: 10.1038/onc.2012.494

61. MacVicar T, Langer T. OPA1 processing in cell death and disease - the long and short of it. J Cell Sci (2016) 129:2297-306. doi: 10.1242/jcs.159186

62. Tomkova V, Sandoval-Acuna C, Torrealba N, Truksa J. Mitochondrial fragmentation, elevated mitochondrial superoxide and respiratory supercomplexes disassembly is connected with the tamoxifen-resistant phenotype of breast cancer cells. Free Radic Biol Med (2019) 143:510-21. doi: 10.1016/j.freeradbiomed.2019.09.004

63. Sehrawat A, Samanta SK, Hahm ER, St Croix C, Watkins S, Singh SV. Withaferin Amediated apoptosis in breast cancer cells is associated with alterations in mitochondrial dynamics. Mitochondrion (2019) 47:282-93. doi: 10.1016/j.mito.2019.01.003

64. Hu J, Zhang Y, Jiang X, Zhang H, Gao Z, Li Y, et al. ROS-mediated activation and mitochondrial translocation of CaMKII contributes to Drp1-dependent mitochondrial fission and apoptosis in triple-negative breast cancer cells by isorhamnetin and chloroquine. J Exp Clin Cancer Res (2019) 38:225. doi: 10.1186/s13046-019-1201-4

65. Seo JH, Chae YC, Kossenkov AV, Lee YG, Tang HY, Agarwal E, et al. MFF Regulation of Mitochondrial Cell Death Is a Therapeutic Target in Cancer. Cancer Res (2019) 79:6215-26. doi: 10.1158/0008-5472.CAN-19-1982

66. McCully JD, Cowan DB, Emani SM, Del Nido PJ. Mitochondrial transplantation: From animal models to clinical use in humans. Mitochondrion (2017) 34:127-34. doi: 10.1016/j.mito.2017.03.004

67. Gollihue JL, Rabchevsky AG. Prospects for therapeutic mitochondrial transplantation. Mitochondrion (2017) 35:70-9. doi: 10.1016/j.mito.2017.05.007

68. Chang JC, Chang HS, Wu YC, Cheng WL, Lin TT, Chang HJ, et al. Mitochondrial transplantation regulates antitumour activity, chemoresistance and mitochondrial dynamics in breast cancer. J Exp Clin Cancer Res (2019) 38:30. doi: 10.1186/ s13046-019-1028-z

69. Chang JC, Chang HS, Wu YC, Cheng WL, Lin TT, Chang HJ, et al. Antitumor Actions of Intratumoral Delivery of Membrane-Fused Mitochondria in a Mouse Model of Triple-Negative Breast Cancers. Onco Targets Ther (2020) 13:5241-55. doi: 10.2147/OTT.S238143

70. Yan C, Duanmu X, Zeng L, Liu B, Song Z. Mitochondrial DNA: Distribution, Mutations, and Elimination. Cells (2019) 8:379. doi: 10.3390/cells8040379

71. Taylor RW, Turnbull DM. Mitochondrial DNA mutations in human disease. Nat Rev Genet (2005) 6:389-402. doi: 10.1038/nrg1606
72. Zong WX, Rabinowitz JD, White E. Mitochondria and Cancer. Mol Cell (2016) 61:667-76. doi: 10.1016/j.molcel.2016.02.011

73. Ghosh JC, Siegelin MD, Vaira V, Faversani A, Tavecchio M, Chae YC, et al. Adaptive mitochondrial reprogramming and resistance to PI3K therapy. J Natl Cancer Inst (2015) 107:dju502. doi: 10.1093/jnci/dju502

74. Wheaton WW, Weinberg SE, Hamanaka RB, Soberanes S, Sullivan LB, Anso E, et al. Metformin inhibits mitochondrial complex I of cancer cells to reduce tumorigenesis. Elife (2014) 3:e02242. doi: 10.7554/eLife.02242

75. Andrzejewski S, Gravel SP, Pollak M, St-Pierre J. Metformin directly acts on mitochondria to alter cellular bioenergetics. Cancer Metab (2014) 2:12. doi: 10.1186/2049-3002-2-12

76. Lee J, Yesilkanal AE, Wynne JP, Frankenberger C, Liu J, Yan J, et al. Effective breast cancer combination therapy targeting $\mathrm{BACH} 1$ and mitochondrial metabolism. Nature (2019) 568:254-8. doi: 10.1038/s41586-019-1005-x

77. Sansone P, Savini C, Kurelac I, Chang Q, Amato LB, Strillacci A, et al. Packaging and transfer of mitochondrial DNA via exosomes regulate escape from dormancy in hormonal therapy-resistant breast cancer. Proc Natl Acad Sci U S A (2017) 114:E9066-75. doi: 10.1073/pnas.1704862114

78. Bajor M, Graczyk-Jarzynka A, Marhelava K, Kurkowiak M, Rahman A, Aura C, et al. Triple Combination of Ascorbate, Menadione and the Inhibition of Peroxiredoxin-1 Produces Synergistic Cytotoxic Effects in Triple-Negative Breast Cancer Cells. Antioxid (Basel) (2020) 9:320. doi: 10.3390/antiox9040320

79. Bohl L, Guizzardi S, Rodriguez V, Hinrichsen L, Rozados V, Cremonezzi D, et al. Combined calcitriol and menadione reduces experimental murine triple negative breast tumor. BioMed Pharmacother (2017) 94:21-6. doi: 10.1016/ j.biopha.2017.07.058

Conflict of Interest: The authors declare that the research was conducted in the absence of any commercial or financial relationships that could be construed as a potential conflict of interest.

Copyright (c) $2021 \mathrm{Li}$ and $\mathrm{Li}$. This is an open-access article distributed under the terms of the Creative Commons Attribution License (CC BY). The use, distribution or reproduction in other forums is permitted, provided the original author(s) and the copyright owner(s) are credited and that the original publication in this journal is cited, in accordance with accepted academic practice. No use, distribution or reproduction is permitted which does not comply with these terms. 